\title{
Laboratorio de enseñanza de energías limpias: el caso de la Universidad Simón Bolívar y su impacto en la comunidad Camurí Grande - Anare (Venezuela)
}

\section{Clean energies teaching laboratory: the case of the Simon Bolivar University and its impact on the Camurí Grande community - Anare (Venezuela)}

\section{Laboratório de ensino de energias limpas: o caso da Universidad Simón Bolívar e seu impacto sobre a comunidade Camurí Grande - Anare (Venezuela)}

\author{
Martín Enrique Durán-García ${ }^{1}$, Emilse Emperatriz Durán-Aponte $^{2}$
}

Forma de citar: M. E. Durán-García, E. E. Durán-Aponte, "Laboratorio de enseñanza de energías limpias: el caso de la Universidad Simón Bolívar y su impacto en la comunidad Camurí Grande - Anare (Venezuela)", Respuestas, vol. 21, no. 1, pp. 16-27, 2016.

Recibido:

Julio 15 de 2015

Aceptado:

Octubre 13

de 2015

\section{Resumen}

El objetivo de esta investigación es diseñar las prácticas del laboratorio de enseñanza de energías limpias enfocadas en el proceso de biodigestión de residuos y desechos sólidos orgánicos que se generan en la comunidad Camurí Grande - Anare, como una estrategia que permite comprender a los estudiantes universitarios de tecnología, el diseño de sistemas útiles de aprovechamiento de estos residuos generados bajo un enfoque tecnológico y social. El método es analítico y comprende la definición de los eventos (aspectos fundamentales y objetivo), la construcción y aplicación de la matriz de análisis (formulación de las prácticas del laboratorio tomando en cuenta todas sus dimensiones), los análisis y conclusiones donde se explican las prácticas formuladas relacionadas con el caso de estudio y sus implicaciones considerando todas las dimensiones desde un enfoque sistémico. Los resultados obtenidos se traducen en el diseño de las prácticas del laboratorio de enseñanza de energías limpias que principalmente comprenden la clasificación y caracterización del biogás producido en términos de eficiencia energética, las pruebas a escala de cinco vivienda escogidas que permiten establecer cuál es el proceso de biodigestión más adecuado para la producción de biogás que garantice la mitigación ambiental y abastecimiento energético, y la transferencia de esta tecnología sostenible fundamentada principalmente en la formación de los estudiantes universitarios y miembros de la comunidad. Conclusión: la formulación de las prácticas del laboratorio de energías limpias bajo un enfoque de sostenibilidad y aprendizaje significativo basado en la cooperación, que permiten ofrecer una solución integral que contribuye con el desarrollo sostenible de la comunidad, y permite a los estudiantes de las carreras tecnológicas de la USB Sede del Litoral desarrollar prácticas de laboratorio donde utilizan un sistema tecnológico como solución tecnológica-ambiental y social a la comunidad.

Palabras Clave: Aprovechamiento energético, biogás, energías limpias, impacto ambiental, laboratorio de enseñanza.
Abstract
The objective of this research was to design the practices for the teaching laboratory on clean energies, focused on the process of bio-digestion of organic solid waste generated 
in the Camurí Grande community- Anare, as a strategy to allow technology-college students to understand the design of useful systems of utilization of these wastes under a technological and social approach. The method is analytical and includes the definition of the events (basic aspects and objective), construction and implementation of analysis matrix (development of laboratory practices taking into account all its dimensions), analyzes and conclusions where the performed practices related to the subject and their implications are explained considering all the dimensions from a systemic approach. The results are translated into the design of the clean energies teaching laboratory practices, mainly including the classification and characterization of the biogas produced in terms of energetic efficiency, scale testing of five selected houses that allow to establish the most adequate digestion process for production of biogas to ensure environmental mitigation and energy supply, as well as the transfer of this sustainable technology based mainly on the training of university students and community members. Conclusion: practices for the teaching laboratory of clean energies were formulated under a cooperative approach based on sustainability and meaningful learning, which can offer a comprehensive solution that contributes to the sustainable development of the community, and allows students of technological careers at USB Sede del Litoral to develop laboratory practices using a technological system as a technological, environmental and social solution to the community.

Keywords: energy efficiency, biogas, clean energy, environmental impact, teaching laboratory.

\section{Resumo}

Objetivo: esta pesquisa teve como objetivo desenvolver práticas de laboratório de ensino de energias limpas focado no processo de biodigestão de resíduos e de desperdícios sólidos orgânicos gerados pela comunidade Camurí Grande - Anare, como uma estratégia que permita aos estudantes universitários de tecnologia compreender o design de sistemas úteis de aproveitamento destes resíduos gerados sob uma abordagem tecnológica e social. Método: foi analítico e compreendeu a definição dos eventos (aspectos fundamentais e objetivo), a construção e aplicação da matriz de análise (formulação das práticas de laboratório tomando em conta todas suas dimensões), os análises e conclusões onde se explicam as práticas formuladas relacionadas com o caso de estudo e suas implicações considerando todas as dimensões desde um enfoque sistémico. Resultados: foi possível o design das práticas de laboratório de ensino de energias limpas, que principalmente compreendeu a classificação e caracterização do biogás produzido em termos de eficiência energética. Os testes a escala de cinco moradias escolhidas permitiram estabelecer qual foi o processo de biodigestão mais adequado para a produção de biogás que pudesse garantir a mitigação ambiental e o abastecimento energético, assim como a transferência desta tecnologia sustentável fundamentada principalmente na formação dos estudantes universitários e membros da comunidade. Conclusão: a formulação das práticas de laboratório de energias limpas sob uma abordagem de sustentabilidade e aprendizagem significativo baseado na cooperação, permite oferecer uma solução integral que contribui com o desenvolvimento sustentável da comunidade, além de permitir aos estudantes dos cursos tecnológicos da USB - Campus do Litoral, desenvolver práticas de laboratório onde utilizam um sistema tecnológico como solução tecnológica-ambiental e social à comunidade.

Palavras-chave: aproveitamento energético, biogás, energias limpas, impacto ambiental, laboratório de ensino. 
Laboratorio de enseñanza de energías limpias: el caso de la Universidad Simón Bolívar y su impacto en la comunidad Camurí Grande - Anare (Venezuela)

\section{Introducción}

Las energías limpias contribuyen con el desarrollo sostenible y equilibrio energético del ambiente, entendiéndose las mismas como aquella que no genera residuos y desechos al generarse. Particularmente en zonas aisladas la problemática de suministro y distribución continua de energía constituye un motivo de no equidad en el acceso de la misma a los ciudadanos que viven en estas comunidades de difícil acceso [1]. La generación de sistemas de producción de energías limpias se ha desarrollado previamente bajo la perspectiva de sostenibilidad, donde se integra la dimensión social, cultural, política, económica, ambiental y técnica que impulsan el desarrollo sustentable de un espacio o colectivo.

Se ha trabajado en sistemas de producción de biocombustibles a partir del aprovechamiento de residuos y desechos sólidos con fines energéticos, permitiendo la satisfacción de necesidades básicas de comunidades aisladas en cuanto al abastecimiento de energía en forma de electricidad y biogás, como la mitigación de pasivos ambientales generados por estos desechos [1]-[2].

Específicamente se ha caracterizado el biogás en pequeños sistemas a escala de laboratorio a partir de la biodigestión de desechos orgánicos vegetales [1], a su vez se ha construido equipos con materiales que se les ha dado un segundo uso, con fines de estimación del poder combustión del biogás producido [2], se ha realizado documentos que establecen la caracterización, concientización, impacto y aprovechamiento de los residuos y desechos sólidos en zonas rurales de difícil acceso [3], [4] y [5], como algunos aportes ante la problemática de preservación del ambiente y aprovechamiento de los recursos que tenemos alrededor de las comunidades.

Por lo tanto, las energías limpias contribuyen significativamente con el desarrollo sostenible y equilibrio energético. Particularmente en zonas aisladas la problemática de suministro y distribución continua constituye un motivo de no equidad en el acceso a la energía para los habitantes de estas zonas de difícil acceso. Es importante entonces que se formen estudiantes universitarios con sólida sensibilidad social que investiguen y desarrollen específicamente en diferentes sistemas de biodigestión para la producción de biogás a partir de las diferentes configuraciones de residuos y desechos sólidos orgánicos que se generen en los diferentes puntos geográficos escogidos. Lo que permite la construcción del biodigestor más adecuado o el gasificador más idóneo desde el punto de vista de eficiencia del sistema y economía de los materiales de construcción basado en el reciclaje, aprovechamiento y uso secundario de los mismos que a su vez contribuye con la minimización de los pasivos ambientales [3], [4], [5], [6], [7].

El estado Vargas cuenta con condiciones y características ambientales disponibles para el aprovechamiento de desechos y residuos sólidos generados por las comunidades y su entorno comercial en general. Todos estos antecedentes permiten verificar la experiencia previa de investigación en el área, y se considera el momento justo para contribuir con las necesidades de los habitantes que viven en zonas aisladas, de manera que aprovechen los desechos orgánicos que generan y concienticen la necesidad de buscar soluciones sencillas que mejoren su condición de vida.

Por lo tanto, el objetivo de esta investigación es diseñar las prácticas del laboratorio de enseñanza de energías limpias enfocadas en el proceso de biodigestión de residuos y desechos sólidos orgánicos que se generan en las comunidad aledaña a la Universidad Simón Bolívar Sede del Litoral (eje Camurí Grande - Anare), como una estrategia que permite comprender a los estudiantes universitarios de tecnología mecánica y demás carreras 
tecnológicas conexas, el diseño de sistemas útiles de aprovechamiento de estos residuos generados en zonas aisladas bajo un enfoque tecnológico y social.

A partir de este hecho, se considera de vital importancia contribuir por ejemplo con el desarrollo del perfil de los futuros Técnicos Superiores de Tecnología Mecánica de la Universidad Simón Bolívar, el cual se fundamenta en la participación activa en las fases que van desde el diseño hasta la operación y mantenimiento de sistemas mecánicos; situación similar con los futuros profesionales en Tecnología Eléctrica, Mantenimiento Aeronáutico y Tecnología Electrónica [6]-[7].

En general, al enseñarse en el área de las ciencias básicas, ciencias técnicas e ingeniería, y en específico a estudiantes universitarios a nivel de técnico superior, se considera la circunscripción de transmisión de conocimientos técnicos, teóricos con cierto enfoque práctico, pero prácticamente desligado y no integrado a las otras áreas del conocimiento [6].

Más aún, los conceptos teóricos y técnicos sólo se relacionan con problemas globales $\mathrm{y}$ abstractos sobre equipos o concepciones industriales donde el futuro profesional se desempeñará profesionalmente, dejando a un lado las interrelaciones entre las diferentes áreas y la concepción técnica impartida, como la relación de la práctica como escenario de aplicación y encuentro con realidades que serán familiares en el ámbito profesional.

La literatura reporta que los estudiantes universitarios tienen diferencias particulares en cuanto al dominio, conceptualización y resolución de problemáticas, que se fundamentan en la rapidez para asimilar un conocimiento, el estilo de aprendizaje y la capacidad para integrar un conocimiento nuevo y relacionarlo con los ya adquiridos previamente [8], [9], [10], [11], [12], [13].
Tomando en cuenta estos aspectos, se debe describir apropiadamente el uso de modelos que contribuyan con el proceso de enseñanza- aprendizaje que permita el mejor aprovechamiento del laboratorio de energías limpias en estudiantes de tecnología industrial de la Universidad Simón Bolívar. Por lo tanto, [6], [7], [8], [12], [14] proponen que el estudiante aprenda a través del aprendizaje significativo, como herramienta que sustituye a la memorización de contenidos y mejora el clima relacional entre el docente y el alumno, al presentarse un ambiente propicio de comprensión, construcción, aprovechamiento y aplicación de diferentes realidades en el tema que se enseña y se aprende, como una solución efectiva en este proceso.

La propuesta de las prácticas del laboratorio de energías limpias se realiza bajo la concepción del aprendizaje significativo a través de la cooperación entrelos diferentes aprendices [9], [11], [12], que en principio son los estudiantes de las carreras tecnológicas del área industrial de la Universidad Simón Bolívar Sede del Litoral, pero que posteriormente puede extenderse a los miembros de la comunidad circundante, con la finalidad de adiestrarlos integralmente en cuanto al uso de este tipo de tecnologías de sencillo acceso.

Las prácticas asociadas al laboratorio, se diseñan de manera que el estudiante y miembros de la comunidad puedan integrar las características del aprendizaje significativo como respuesta a problemas reales, como el de la generación de residuos y desechos sólidos al ambiente. Se configuran conceptos técnicos asociados a la termodinámica, mecánica, gestión ambiental y de riesgo, responsabilidad social, conciencia ecológica, entre otros, para resolver a través de procedimientos principalmente técnicos la problemática de mitigación de pasivos ambientales de manera que se convierta en una oportunidad para el aprovechamiento energético correspondiente.
Enero - Junio 2016 ISSN 0122-820X E-ISSN 2422-5053 PP: 16-27 
Laboratorio de enseñanza de energías limpias: el caso de la Universidad Simón Bolívar y su impacto en la comunidad Camurí Grande - Anare (Venezuela)
Por lo tanto, el aprendizaje significativo a través de la cooperación asume cinco aspectos que con el tiempo los diferentes aprendices adoptarán de forma implícita propuestos por [7]:

Interdependencia positiva donde el instructor propone una asignación clara y objetivo grupal como alcance que los estudiantes deben validar.

La responsabilidad individual y grupal que debe ser asumida para alcanzar las metas particulares y compartidas de acuerdo al plan de trabajo trazado.

La interacción estimuladora que permita una labor que promueva positivamente el éxito de los compañeros de grupo en cada práctica.

Las prácticas interpersonales y grupales como actividad que permite el adecuado funcionamiento del trabajo en equipo.

La evaluación grupal que permite medir el alcance de las metas siempre conservando el trabajo eficaz y positivo.

Este esquema de trabajo durante las prácticas de laboratorio, permite que se desarrollen competencias como escuchar, aceptar sugerencias, confiar en las respuestas de los demás miembros del grupo, administrar el tiempo, autoevaluar el desempeño, tolerar, alcanzar el consenso y compromiso con la asignación dada. Estas competencias contribuyen con la formación integral del futuro profesional egresado de la universidad [6] y la formación de los miembros de la comunidad como contribución al desarrollo humano y sostenible, lo cual apoya al objetivo 7 para el desarrollo del milenio que busca garantizar la sostenibilidad del ambiente [15], [16].

\section{Materiales y métodos}

El método muestra la forma como se estructura la investigación, como se controla sus resultados, de qué forma se presenta las posibles soluciones como respuesta a la problemática que se tiene. Por lo tanto el conjunto de actividades a realizarse buscan la concreción del objetivo planteado. El método utilizado es analítico según [17] donde se intenta comprender las situaciones en términos de las relaciones entre sus componentes, descubrir cada elemento e identificar las sinergias menos evidentes de los analizados.

Las fases del método analítico son la definición de los eventos, construcción de la matriz de análisis, aplicación de la matriz de análisis, el análisis y conclusiones, con el fin último de diseñar las prácticas asociadas al laboratorio de energías limpias que permita descubrir los elementos no visibles en el proceso investigativo planteado, tal como se muestra en la Figura 1.

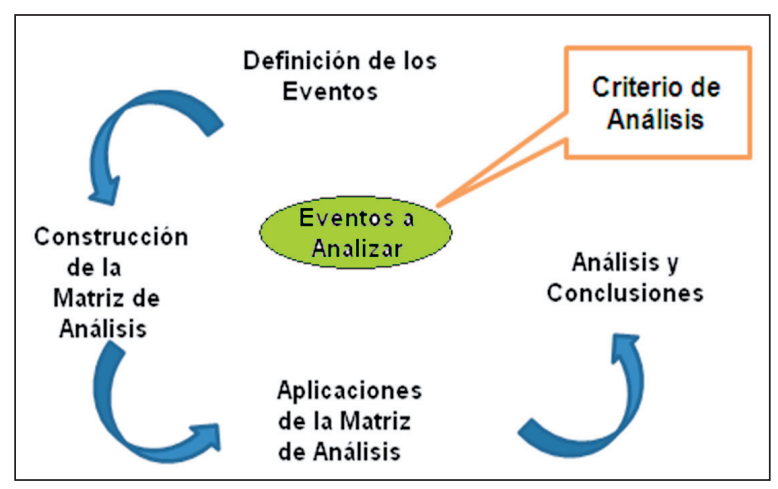

Figura 1. Esquema de una Investigación Analítica Fuente: [17].

La definición de los eventos corresponde a la definición de los aspectos fundamentales, objetivo y de los principios más importantes de la problemática planteada, en la construcción de la matriz de análisis y aplicación de la matriz se formulan las prácticas del laboratorio de energías limpias tomando en cuenta todas sus dimensiones, mientras que en el análisis y conclusiones se explican las implicaciones de las prácticas del laboratorio formulado de manera que permita identificar la adecuación plena del caso de estudio en el ambiente seleccionado, garantizando el máximo aprovechamiento de aprendizaje a través de la formulación e implementación 
de las mismas en el laboratorio de energías limpias, encontrándose a su vez los resultados no visibles que describen a todo el proceso. Por lo tanto, la producción de biogás medida en términos de eficiencia energética o rendimiento se considera de los resultados obtenidos en [1], [5].

\section{Resultados y Análisis}

La formulación de las prácticas del laboratorio de energías limpias en la Universidad Simón Bolívar se fundamenta en un conjunto de actividades las cuales tienen fundamento en las investigaciones previas realizadas por [1], [2], [5], [17], [18], [19], [20], [21], las cuales permiten comprender los valores cuantitativos asumidos en cuanto a la producción de biogás en términos de rendimiento, eficiencia energética entre otros:

- Inventario de los residuos y desechos sólidos generados en las zonas aisladas del eje Camurí - Anare con el propósito de regionalizar la generación de estos desechos.

- Clasificación de las muestras tomadas en función de ternas o agrupaciones mayores de desechos y residuos generados previa separación de las mismas.

- Generación de las combinaciones de los residuos y desechos sólidos que permita verificar la cantidad de biocombustible (metano) que se forma, y realizar las comparaciones correspondientes en cuanto a producción y rendimiento.

En la Figura 2, se representa el resultado de algunas actividades de estas primeras prácticas a través del diseño de probetas PET (polietilen tereftalato) de 0,6 litros en el proceso de conversión de residuos y desechos sólidos orgánicos vegetales llamado biodigestión anaeróbica para la producción de biogás, específicamente con el fin de generar el inventario, clasificar las muestras y generar las combinaciones de estos desechos [1].

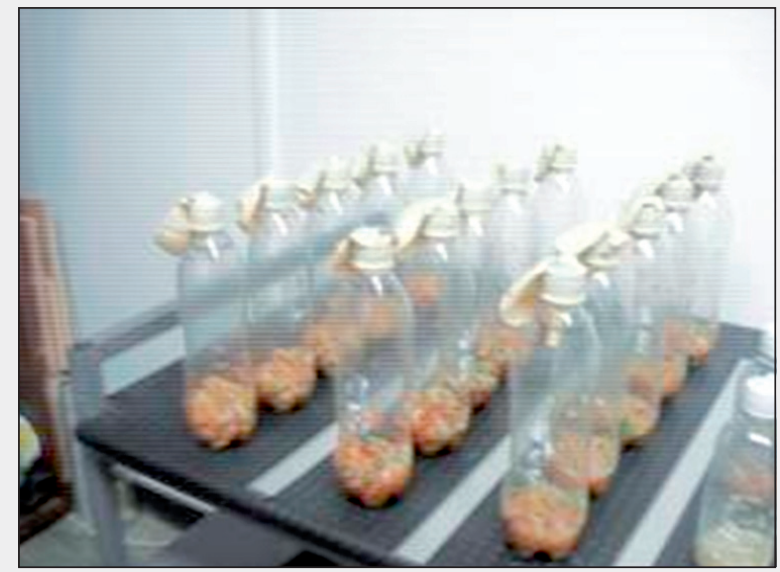

Enero - Junio 2016 ISSN 0122-820X E-ISSN 2422-5053 PP: 16-27

Figura 2. Probetas PET de 0,6 litros. Proceso de biodigestión anaeróbica, producción de biogás en probetas PET Fuente: [1]

Estas probetas son contenedores de bebidas gaseosas que han sido desechadas, y se le está dando un segundo uso con fines de aprovechamiento energético a partir de la correspondiente mitigación del pasivo ambiental generado.

- Diseño del equipo de conversión de los residuos y desechos sólidos: biodigestor y gasificador con todas sus conexiones correspondientes. En la Figura 3, se presentan algunos bosquejos del montaje experimental del sistema escalado en su versión preliminar [1], [2], [21], [22].

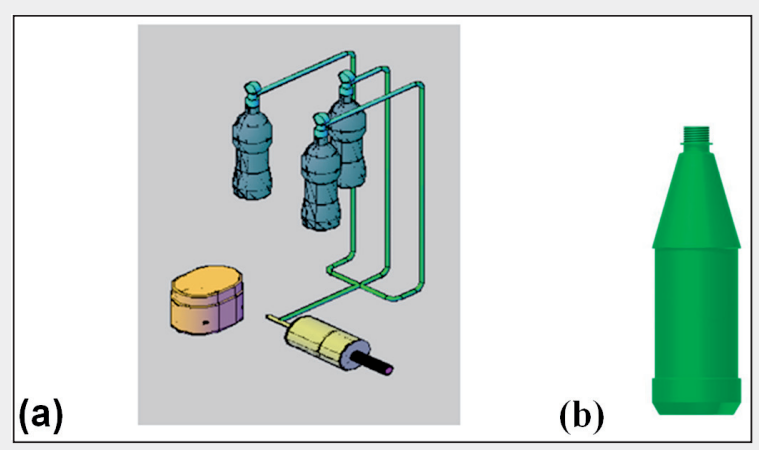

Figura 3. (a) Bosquejo del sistema de tuberías, sistema de trasegado y bombona de almacenamiento del proceso de producción de biogás en probetas PET, (b) Prototipo probetas PET

Fuente: [21]

- Pruebas a pequeña escala de los equipos de conversión de los residuos y desechos sólidos a partir de las diferentes configuraciones de alimentación 
Laboratorio de enseñanza de energías limpias: el caso de la Universidad Simón Bolívar y su impacto en la comunidad Camurí Grande - Anare (Venezuela)

seleccionadas [1], [5], [21], [22].

El escalamiento del sistema de conversión de los residuos y desechos sólidos, se establece principalmente al tomar en cuenta los recursos disponibles, el espacio físico a utilizar, el objetivo a cumplir energéticamente, la sostenibilidad energética y ambiental, y la factibilidad de controlar variables termodinámicas del sistema que está plenamente influenciado por su entorno.

- Caracterización del biocombustible producido en términos de eficiencia energética a través de un calorímetro de Junkers.

En cuanto al proceso de valoración energética del proceso de producción de biogás en probetas PET se construyó un sistema de calorimetría propuesto por [2] tal como se muestra en la Figura 4. Es importante destacar que los costos de construcción y ensamblaje de todas las partes del sistema de calorimetría es bajo no excediendo de los diez dólares americanos (10 \$), en virtud que algunos materiales se les da un segundo uso funcionalmente una vez que han sido desechados originalmente.

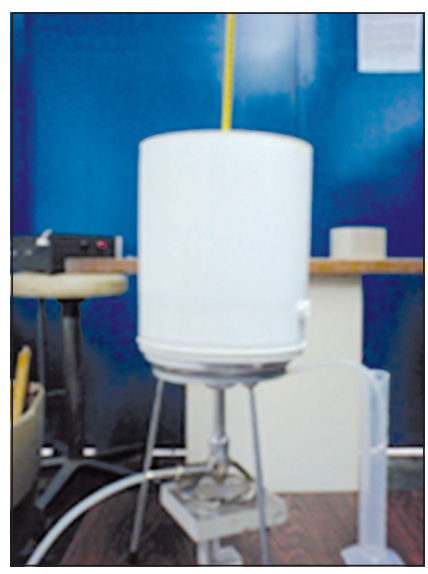

Figura 4. Sistema de Calorimetría: Calorímetro tipo Junkers. Fuente: Autor con base en [2]

- Caracterización del biocombustible producido en términos de composición a través de un cromatógrafo de gases [2]. En esta práctica los estudiantes y miembros de la comunidad realizan todas las actividades normas de seguridad y uso del equipo, reciben las instrucciones del técnico correspondiente, toman la muestra de biogás, adecuación de la misma para que sean analizadas en el cromatógrafo, la toma y adecuación de los resultados, y su respectiva repetitividad.

- Pruebas piloto a escala de cinco viviendas escogidas de las zonas aisladas del eje Camurí - Anare [21].

El papel orientador de la psicología educativa, el trabajo social, la sociología, entre otras disciplinas es importante en esta práctica, en virtud que intervienen activamente en la adaptación y concientización del nuevo grupo usuarios, estudiantes de carreras tecnológicas industriales de la Universidad Simón Bolívar y especialmente miembros de la comunidad de las zonas aisladas en estudio, para el uso de la tecnología de conversión de residuos y desechos sólidos y sus procesos conexos.

Particularmente, se ha seguido el protocolo de enseñanza aprendizaje conforme a lo propuesto por [3], [7], [8], [9], [10], donde se busca a través de las prácticas integrar el aprendizaje cooperativo, prácticas profesionales y competencias sociales en los estudiantes y miembros de la comunidad.

Los criterios de selección de estas viviendas corresponde a los reflejados en los resultados previos de un proyecto de energías limpias propuesto por [5], [21] donde se toma en cuenta los requerimientos energéticos promedio de la vivienda unifamiliar en zona aislada (luz y gas principalmente), tamaño del grupo familiar, estilo de alimentación, conservación de alimentos, y el posible abastecimiento de la demanda energética (80\% de los requerimientos de la vivienda) [5] a través del sistema de energía limpia diseñado (biodigestor o gasificador) satisfaciendo en parte los objetivos 8 y 12 de desarrollo del milenio [16]. 
- Clasificación de los tipos de biocombustibles producidos de acuerdo a su composición química y fuentes de origen [4]. Comprende las actividades de contraste y comparación de las muestras tomadas con los reportados históricamente en el laboratorio y en la literatura.

Tabla I. Cronograma de actividades a desarrollarse en el laboratorio de energías limpias.

\begin{tabular}{|c|c|c|c|c|c|c|c|c|c|c|c|c|c|}
\hline \multirow{2}{*}{ Objetivos específicos } & \multirow{2}{*}{ Actividad } & \multicolumn{12}{|c|}{ Semanas } \\
\hline & & 1 & 2 & 3 & 4 & 5 & 6 & 7 & 8 & 9 & 10 & 11 & 12 \\
\hline \multirow{2}{*}{$\begin{array}{l}\text { Estudiar la generación de desechos } \\
\text { sólidos orgánicos en tres zonas aisladas } \\
\text { del eje Camurí - Anare }\end{array}$} & $\begin{array}{l}\text { Realizar un inventario de los desechos sólidos } \\
\text { orgánicos que se generan en las zonas aisladas del } \\
\text { eje Camurí - Anare }\end{array}$ & $\mathbf{X}$ & $\mathrm{X}$ & & & & & & & & & & \\
\hline & $\begin{array}{l}\text { Clasificar y generar las combinaciones de las } \\
\text { muestras tomadas en función de ternas o } \\
\text { agrupaciones mayores de residuos generados previa } \\
\text { separación de las muestras tomadas. }\end{array}$ & & & $\mathbf{X}$ & $\mathrm{X}$ & & & & & & & & \\
\hline \multirow{3}{*}{$\begin{array}{l}\text { Caracterizar y diseñar los sistemas de } \\
\text { conversión de los residuos y desechos } \\
\text { sólidos que se ajustan a las } \\
\text { comunidades aisladas del eje Camurí - } \\
\text { Anare }\end{array}$} & $\begin{array}{l}\text { Diseñar el equipo de conversión de los residuos y } \\
\text { desechos sólidos, y realizar pruebas a pequeña } \\
\text { escala a partir de las diferentes configuraciones de } \\
\text { residuos sólidos orgánicos seleccionados. }\end{array}$ & & & & $\mathbf{X}$ & $\mathrm{X}$ & $\mathbf{X}$ & $\mathbf{X}$ & $\mathbf{X}$ & $\mathrm{X}$ & & & \\
\hline & $\begin{array}{l}\text { Caracterizar el biocombustible producido en } \\
\text { términos de eficiencia energética y composición a } \\
\text { través de los equipos correspondientes }\end{array}$ & & & & & & & & & & $\mathbf{X}$ & $\mathbf{X}$ & \\
\hline & $\begin{array}{l}\text { Realizar pruebas a escala de cinco vivienda } \\
\text { escogidas de las zonas aisladas del eje Camurí - } \\
\text { Anare }\end{array}$ & & & & & & & & & & & $\mathbf{X}$ & $\mathbf{X}$ \\
\hline
\end{tabular}

Fuente: Autor

Estas actividades se enmarcan dentro cinco grandes actividades que se desarrollan a lo largo de un período lectivo de la Universidad Simón Bolívar, el cual consta de 12 semanas del calendario académico donde realizarán todas la prácticas tanto a los estudiantes de la universidad como a los miembros de la comunidad, las cuales se realizan en el Laboratorio $\mathrm{G}$ donde se disponen de los equipos, instalaciones y materiales correspondientes, el personal docente y técnico de laboratorio, y el espacio para el desarrollo de cada práctica de laboratorio en todas sus fases.

En especial, los miembros de la comunidad serán capacitados en la primera semana de cada una de las 5 actividades que abarcan todas las prácticas del laboratorio, de manera que se entrenen y comprendan los principios básicos
A continuación se presenta la tabla I la cual describe las actividades desarrolladas en función de los objetivos específicos que contribuyen con la realización de las prácticas del laboratorio de energías limpias. tecnológicos y ambientales de los sistemas de conversión biológica (biodigestor o gasificador).

Los estudiantes serán evaluados conforme al sistema de evaluación formal de la Universidad Simón Bolívar, mientras que los miembros de la comunidad realizarán sus actividades con asesoría directa del personal técnico y profesional, cuya evaluación corresponde al adiestramiento del manejo de este sistema de producción de energía limpia que se traduce en la obtención de mejores resultados en cuanto a producción de biogás y mitigación de pasivos ambientales en cuanto a la generación cotidiana de residuos y desechos sólidos. ISSN 0122-820X -ISSN 2422-5053 PP: 16-27

\section{.}

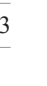


Laboratorio de enseñanza de energías limpias: el caso de la Universidad Simón Bolívar y su impacto en la comunidad Camurí Grande - Anare (Venezuela)

Tabla II. Plan de inversión en el laboratorio de energías limpias.

\begin{tabular}{|c|c|c|}
\hline Descripción & Propósito & Unidad \\
\hline Computadora & $\begin{array}{l}\text { Procesamiento de datos experimentales, conexión con } \\
\text { software de otros equipos }\end{array}$ & 02 \\
\hline impresora Multifuncional & $\begin{array}{l}\text { Generar los reportes de experimentación, escanear } \\
\text { documentos para los experimentos }\end{array}$ & 01 \\
\hline Portátil & Soporte de software para trabajo en campo & 01 \\
\hline Fotocopiadora y escáner & Reproducir reportes de la experimentación & 01 \\
\hline Sistema de Cromatografia de Gases & Análisis de gases combustibles generados & 01 \\
\hline Sistema de Extracción de Gases & Campana de gases para manejo de las muestras & 01 \\
\hline Sistema de calorimetría & $\begin{array}{l}\text { Estimar el poder calorífico y eficiencia energética del } \\
\text { sistema de gases }\end{array}$ & 01 \\
\hline Sistemas de presurización y estimación de temperatura & Monitorear todos los procesos de experimentación & 01 \\
\hline Sistemas de control de procesos & Control de las variables termodinámicas & 02 \\
\hline Sistema de mobiliario para experimentación de gases & $\begin{array}{l}\text { Soporta y protege a los equipos, además que genera la } \\
\text { estructura adecuada para la experimentación }\end{array}$ & 01 \\
\hline Mesa de escritorio & $\begin{array}{l}\text { Estación de trabajo y soporte para las computadoras y } \\
\text { fotocopiadora }\end{array}$ & 05 \\
\hline Sillas ejecutivas de escritorio & para la estación de trabajo & 04 \\
\hline Archivadores y estantes & $\begin{array}{l}\text { archivo de secuencia de documentos y reportes de los } \\
\text { experimentos }\end{array}$ & 05 \\
\hline Cartelera de madera y vidrio & Información de todo el proceso & 02 \\
\hline Sistema de vidriería de química de gases & $\begin{array}{l}\text { todas las estructuras, partes y accesorios necesarios para la } \\
\text { experimentación }\end{array}$ & 01 \\
\hline $\begin{array}{l}\text { Mobiliario: Mesas, sillas, estantería, vidriería y } \\
\text { cartelera }\end{array}$ & Soporte de todas las actividades del laboratorio & 01 \\
\hline Servicios: albañilería y herrería. & $\begin{array}{l}\text { Reacondicionamiento y seguridad de todo el espacio físico } \\
\text { del laboratorio }\end{array}$ & 01 \\
\hline Sistema de Biodigestores de 0,5 litros & $\begin{array}{l}\text { Conversión biológica de una muestra a escala laboratorio } \\
\text { de residuos y desechos sólidos }\end{array}$ & 15 \\
\hline Sistema de Biodigestores de 5 litros & $\begin{array}{l}\text { Conversión biológica de una muestra de residuos y } \\
\text { desechos sólidos a escala banco (vivienda unifamiliares) }\end{array}$ & 05 \\
\hline
\end{tabular}

Fuente: Autor

Convertir los retos en nuevas potencialidades ambientales, socioeconómicas y tecnológicas, no sólo es un requisito de sostenibilidad, sino de competitividad, habitabilidad y de estabilidad a largo plazo. La inversión total del laboratorio fue de $5500 \$$, cantidad que en principio no representa un monto manejable para la Universidad en cuanto al carácter deficitario de su presupuesto se refiere, sin embargo gracias a los esfuerzos y sinergias mediante el cofinanciamiento con la empresa privada se ha podido configurar el laboratorio de enseñanza de energías limpias.

Sin embargo, dentro del concepto de la sostenibilidad constituye un reto la formulación y diseño de las prácticas del laboratorio en términos que:

- Se convierte en un espacio de aprendizaje significativo bajo la premisa de la cooperación en función del trabajo en equipo para los estudiantes de carreras tecnológicas como para los miembros de la comunidad.

- Contribuye con la solución de un problema de creciente generación de pasivos ambientales en la zona aislada de Camurí - Anare.

- Se capacita a la población en el manejo de 
estos sistemas de conversión de residuos y desecho sólidos.

- Se abastece de energía en forma de biocombustible con fines de uso doméstico una vez que los miembros de las comunidades se hallan entrenado en el laboratorio. Esto se refleja a partir de la actividad 3, donde los miembros de la comunidad pueden construir su sistema de energía limpia en sus casas.

- La instalación del laboratorio se asume por instituciones privadas, $\mathrm{ONG}$ e instituciones públicas interesadas en invertir en la ejecución de este tipo de proyecto en todos sus niveles $\mathrm{y}$ dimensiones.

- Se adecuan los espacios que permitan el montaje en todas sus fases y dimensiones de la tecnología de conversión de residuos y desechos sólidos.

- Se formalizan espacios para procesos de producción del biocombustible a generar.

- Se establecen nuevos responsables que administraran los espacios e instalaciones correspondientes a las viviendas unifamiliares ubicadas en zonas aisladas.

- Se documentan todos estos cambios y adaptaciones.

- Se genera la documentación que soporta las diferentes actividades como negociación de la tecnología, el funcionamiento de los equipos, los diagramas de los subprocesos, el establecimiento de los procesos, requerimientos y especificaciones, servicios y sistemas de control.

Esto representa una gran oportunidad donde que se fundamenta en una nueva manera de abordar la gestión de aprovechamiento de los recursos que se consideran residuos y desechos sólidos, en medio de nuevas tecnologías sostenibles que aporten soluciones integrales al ambiente [3], [15], [21]. Es una solución integral pues se aborda un problema de abastecimiento energético a través de una solución tecnológica (biodigestor o gasificador), social (en servicio a la comunidad) y ambiental (mitigando la generación y acumulación de residuos y desechos sólidos). A su vez se pueden entender que la remuneración de los miembros de la comunidad seria aprender el adecuado uso de los biodigestores que los abastecen de gran parte de sus necesidades energéticas domésticas y contribuyen con la generación de pasivos ambientales

\section{Conclusiones}

En función de los resultados obtenidos, se considera que el diseño de las prácticas del laboratorio de enseñanza de energías limpias en la Universidad Simón Bolívar, bajo un enfoque de sostenibilidad y aprendizaje significativo basado en la cooperación, constituye una solución integral que contribuye con el desarrollo sostenible de la comunidad aislada asociada al eje Camurí Anare.

Recurrir a un modelo de enseñanza alternativa a la convencional, produce en los estudiantes de las carreras tecnológicas un interés ante lo novedoso, y puede contribuir con el esfuerzo por alcanzar los objetivos planteados durante la ejecución de cada práctica de laboratorio que se realice.

Por lo tanto, los resultados obtenidos además se traducen en la formación de estudiantes universitarios y miembros de la comunidad en un sólido laboratorio de análisis de residuos y desechos sólidos orgánicos que esté al servicio de la comunidad, la clasificación y caracterización el biogás producido en términos de eficiencia energética sostenible, las pruebas a escala de cinco vivienda escogidas de la zona aislada, de manera que
Enero - Junio 2016

ISSN 0122-820X

E-ISSN 2422-5053

PP: 16-27 
Laboratorio de enseñanza de energías limpias: el caso de la Universidad Simón Bolívar y su impacto en la comunidad Camurí Grande - Anare (Venezuela)

se establezca el proceso más adecuado de producción de energía limpia en forma de biogás que garantice la mitigación ambiental y gran parte del abastecimiento energético, y la transferencia de esta tecnología sostenible en la comunidad.

\section{Referencias}

[1] M. Durán-García, Y. Ramírez, L. Rojas, y R. Bravo. "Biogas home-production assessment using a selective sample of organic vegetable waste - A preliminary study". Revista Interciencia, vol. 37, pp. $128-132,2012$.

[2] M. Durán-García, A. Blanco, y L. Pérez. "Prototipo de un sistema de calorimetría con fines de rendimiento energético en la producción de biogás". Revista Venezolana de Tecnología y Sociedad, vol. 6, pp. $11-24,2013$.

[3] M. Durán-García. "Biocombustibles: el respeto a la naturaleza con responsabilidad común". IX Congreso Venezolano Interdisciplinario de Orientación. Memoria de Congreso, Venezuela, Asociación de Orientadores de Venezuela, Venezuela, pp. 1-10, 2012.

[4] M. Durán-García. "Gasificación de la Biomasa Residual: Avances en Bioingeniería". Ingeniería y Ciencias Aplicadas: Modelos Matemáticos y Computacionales. Sociedad Venezolana de Métodos Numéricos en Ingeniería, pp. BSB-7 - BSB-12, 2014.

[5] M. Durán-García. "Gasificación a través de la Biodigestión Anaeróbica de la Biomasa Residual en Zonas Aisladas de Venezuela". XXI Convención Internacional y X Exhibición Industrial del Gas, pp. 1-8, mayo de 2015.

[6] E. Durán-Aponte, y M. Durán-García. "Competencias sociales y las prácticas profesionales. Vivencias y demandas para la formación universitaria actual”. Revista Cultura y Educación, vol. 24, no. 1, pp. 61-76, 2012.

[7] M. Durán-García, y E. Duran-Aponte. "La termodinámica en los estudiantes de tecnología: una experiencia de aprendizaje cooperativo". Revista Enseñanza de las Ciencias, vol. 31, pp. $45-59,2013$

[8] S. Flores, A. Trejo y L. Trejo. “¿Cómo mejorar el proceso enseñanza - aprendizaje mediante la evaluación - regulación? El caso de la termodinámica". Memorias de las Terceras Jornadas Internacionales de la Enseñanza Universitaria de la Química, Argentina, pp. 1-8, 2003.

[9] M. Mendoza. "Grupos cooperativos de aprendizaje en el trabajo tutorial". Revista Innovación Educativa, vol. 5, pp. 47-53, 2005.

[10] L. García. "La formación en la educación técnica universitaria". I Congreso Iberoamericano de Enseñanza de la Ingeniería, CIEI-149. Memoria en Congreso, Venezuela, pp. 1-9, 2009.

[11] M. Nava, X. Arrieta y M. Flores. "Referentes teóricos de una instrucción orientada a la construcción de conceptos científicos en física". Revista de la Facultad de Ingeniería UCV, vol. 24, pp. 98-111, 2009.

[12] R. Oñate y S. Sánchez. "Resolución de problemas por investigación y su influencia en los trabajos prácticos de laboratorio en termodinámica". Revista de Pedagogía, vol. 31, no. 89, pp. 307329, 2010.

[13] E. Duran-Aponte y M. Durán-García. "Aprendizaje cooperativo en la enseñanza de termodinámica: estilos de aprendizaje y atribuciones causales". 
Revista Estilos de Aprendizaje, vol. 11, pp. $256-275,2013$.

[14] J. Martínez y B. Pérez. "Estudio de propuestas alternativas en la enseñanza de la termodinámica básica". Revista Enseñanza de las Ciencias, vol. 15, no. 3, pp. 287-300, 1997.

[15] A. Gabaldón. "El Desarrollo Sustentable. La salida para América Latina". Corporación Andina de Fomento, Grijalbo, Venezuela, 2006.

[16] ONU Asamblea General. Transformar nuestro mundo: la Agenda 2030 para el Desarrollo Sostenible (Objetivos de Desarrollo del Milenio), pp. 1-40, 2015.

[17] J. Hurtado. "El proyecto de investigación". Ediciones Quirón, Venezuela, 2012.

[18] K. Kujawa-Roeleveld, T. Elmitwalli, M. Van Leeuwen, A. Tawfik, T. De Mes y G. Zeeman. "Digestión anaeróbica de residuos fisiológicos y desperdicios de cocina para la gestión de recursos en el sistema DESAR". 2do Simposio Internacional Sobre Saneamiento Ecológico. ECOSAN. Symposium. Luebeck, Alemania, pp. 439-448, 2004

[19] R. Cham y E. Vivanco. "Potencial del biogás: identificación y clasificación de los distintos tipos de biomasa disponibles en chile para la generación de biogás". Proyecto de Energías Renovables y Deutsche Gesellschaft für Technische Zusammenarbeit (GTZ) GMBH (Cooperación Técnica Alemana). Chile, pp. 1-82, 2007.

[20] S. Lansing, J. Víquez, H. Martinez, R. Botero y J. Martin. "Quantifying electricity generation and waste transformations in a low-cost, plug-flow anaerobic digestion system”. Ecological
Engineering, vol. 34, pp. 332-348, 2008.

[21] M. Durán-García, M y E. DuranAponte. "Conceptos de Calor y Trabajo en un foro electrónico. Efectos de la autoeficacia computacional". Revista Educación Química, vol. 24, pp. 247 254, 2013.

[22] J. Figueroa. "Diseño de un banco de prueba para la producción de biogás". Informe de pasantías Técnico Superior Universitario en Tecnología Mecánica, Universidad Simón Bolivar, Venezuela, pp. 1-59, 2011.

Enero - Junio 2016

ISSN 0122-820X

E-ISSN 2422-5053 